\title{
MEMS Microrobot Controlled by Mounted Neural Networks IC with Two Types Actuators
}

\author{
Kei Iwata, Hirozumi Oku, Yuki Okane, Yohei Asano, Masaki Tatani, Yuki Ishihara, Kazuki Sugita, \\ Precision Machinery Engineering, Graduate school of Science and Technology, Nihon University, \\ 7-24-1, Narashinodai, Funabashi, Chiba, 274-8501, Japan \\ Satohiro Chiba, Satoko Ono, Mizuki Abe, Minami Takato, Ken Saito, Fumio Uchikoba \\ Department of Precision Machinery Engineering, College of Science and Technology, Nihon University, \\ 7-24-1, Narashinodai, Funabashi, Chiba, 274-8501, Japan \\ E-mail: takato@eme.cst.nihon-u.ac.jp,kensaito@eme.cst.nihon-u.ac.jp,uchikoba@eme.cst.nihon-u.ac.jp
}

\begin{abstract}
We report the hexapod microrobot controlled by the bare chip IC of hardware neural networks. MEMS (micro electro mechanical systems) technology is used for fabrication of the microrobot. Lead zirconium titanate (PZT) and shape memory alloy (SMA) are used in each actuator, respectively. As the result, PZT type is realized the walking motion by bare chip IC. Moreover, SMA type shows the hexapod walking locomotion with mounted bare chip IC. The walking speed is $2.4 \mathrm{~mm} / \mathrm{min}$ and the step width was $0.083 \mathrm{~mm}$.
\end{abstract}

Keywords: Microrobot, Artificial Neural Networks, MEMS, PZT, SMA.

\section{Introduction}

Insects have small sophisticated motion mechanisms controlled by flexible brain neural networks. Therefore, fully understanding how insects move is among the ultimate goals of microrobotic studies.

Many researches that mimic the flexible motion mechanism of insects have been reported ${ }^{1}$. Especially, walking is a typical movement mechanism of insects. The robot mimicking walking mechanism by using legs has been reported ${ }^{2}$. However, most cases of them are large body compared with the insects. Realization of the small walking mechanism as large as the insect is difficult in conventional process. Therefore, the demand for micro electro mechanical systems (MEMS) technology that can create miniature components with high degrees of accuracy is increasing ${ }^{2}$. In addition, the big feature of the insects is flexible thinking circuit.
The robots that introduce the model of the neural networks as the control system are reported ${ }^{3}$. However, the small size robot controlled by neural networks is rare. Therefore, robots that are perfectly mimicked the insects have not been realized.

Previously, we reported on an insect-type MEMS microrobot that fits within 5-mm-cubed driven by a pulsed hardware neural networks $(\mathrm{P}-\mathrm{HNN})^{4}$. However, the P-HNN circuit, which was constructed from discreet components, was found to be too big to be mounted on the microrobot. We then developed a P-HNN integrated circuit (IC) using a bare chip IC and peripheral amplifying circuits, but the resulting control system was still too $\mathrm{big}^{5}$. To solve the problem, the bare chip IC for SMA (shape memory alloy)-type microrobot was newly developed ${ }^{6}$.

In this paper, we report on the development of insecttype microrobots controlled by bare chip IC of artificial neural networks. Actuators of the microrobots are used piezoelectric element composed of lead zirconium 
titanate (PZT) and artificial muscle wires composed of shape memory alloy. The bare chip ICs for each type of microrobot were newly developed.

\section{Mechanism}

Insect-type microrobot has hexapod legs like insects and falls within 5-mm-cubic. The designs of the two-type microrobot are shown in figure 1. The components of the microrobot are the frame, rotary actuator, and the link mechanisms. PZT and SMA are easy to downsize because the material itself has the function of the actuator. Almost all of the component are fabricated from silicon wafer using MEMS machining technology.

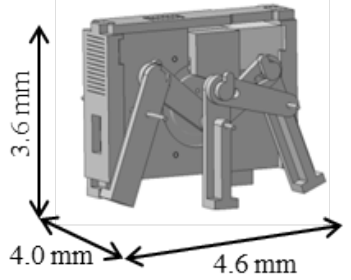

(a)PZT-type

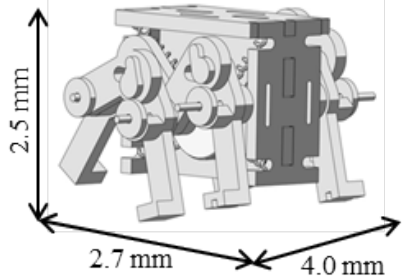

(b)SMA -type
Fig. 1. Microrobot designs.

\subsection{PZT-type microrobot}

The PZT distorts by applying a voltage. In this study, a multilayer piezoelectric element composed of PZT is used for low voltage driving. The PZT has advantage of low electricity consumption and fast response time. However, it is difficult to use PZT as direct driven actuator because of small displacement. Therefore, the impact mechanism is adopted for the actuator of the microrobot. The design of the PZT-type actuator, which has side, end, and height dimensions of 1.0, 4.4, and 3.2 $\mathrm{mm}$, respectively, is shown in figure 2 . The displacements of a multilayer piezoelectric element are $110 \mathrm{~nm}$ at applied $6 \mathrm{~V}$. The impact head is attached to the PZT and adjusted to contact to the impact head. The PZT impacts the rotor to generate rotary motion.

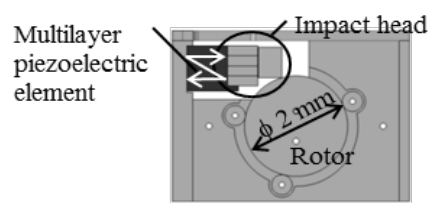

Fig. 2. Mechanism of the PZT-type rotary actuator.

\subsection{SMA-type microrobot}

SMA which shrinks at high temperatures and expands at low temperatures is used for the rotary actuator. The SMA displacement can be changed by applying electrical energy to create heat. We used $0.05 \mathrm{~mm}$ diameter wire to create the SMA with a coil diameter of $0.2 \mathrm{~mm}$. A standard drive current from 50 to $120 \mathrm{~mA}$ was used, and a maximum displacement of $50 \%$ of the original length was achieved. Figure 3 shows the design of SMA-type rotary actuator.

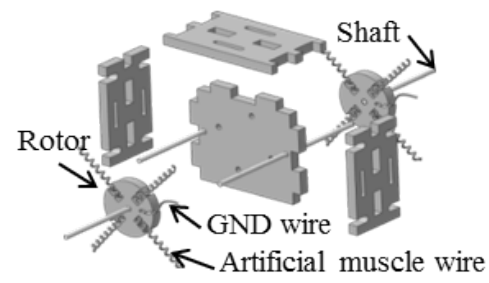

Fig. 3. Mechanism of the SMA-type actuator.

Four SMAs and a ground (GND) wire are connected to the rotor. Rotational motion of the actuator is generated by passing an electric current through the SMA in rotation.

\subsection{Walking mechanism}

Insects perform a stable hexapod walking by grounding the three legs. In this paper, link mechanisms are adopted for realizing hexapod walking like insects. Rotary motion of the actuator is converted into walking motion by link mechanisms via a shaft shown in figure 4. The locomotion pattern uses a $180^{\circ}$ phase shift on each side.

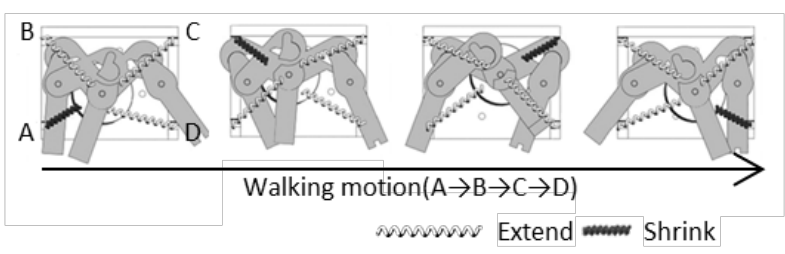

Fig. 4. Walking mechanism of the microrobot.

\section{Control system}

Insects function by using the neural networks in their own brains. In this study, we mimic those neural circuits using an analog system that allows the microrobot to function in an insect-like manner. 


\subsection{Cell body model}

The PZT-type microrobot is controlled by the cell body model shown in figure 5 . The cell body model has refractory period, analog characteristic of the output pulse and time varying negative resistance characteristic. Driving frequencies of the PZT-type microrobot is 25 $\mathrm{kHz}$. The amplifier circuit is added to obtain the output voltage $20 \mathrm{~V}$.

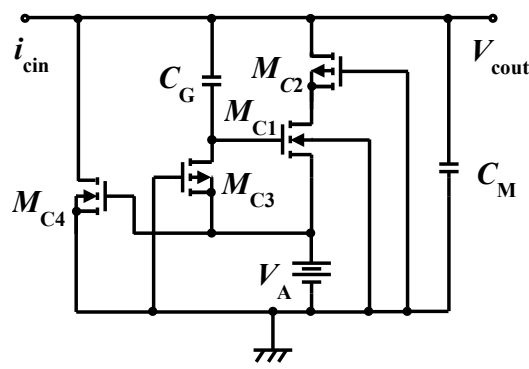

Fig. 5. Cell body model circuit diagram.

\subsection{CPG model}

It is well known that locomotion rhythms of living organisms are generated by central pattern generator (CPG). The SMA-type microrobot is controlled by the CPG model. CPG model can be formed by inhibitory synaptic connection of four neurons shown in Figure 6.

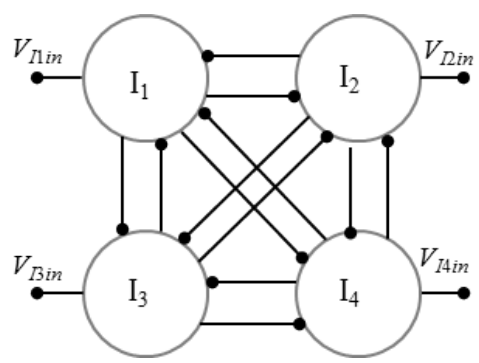

Fig. 6. Artificial neuron circuit connection diagram.

Figure 7 shows the circuit diagram of the neuron composed the cell body model and inhibitory synaptic model. The inhibitory synaptic model has spatiotemporal summation characteristics similar to living organisms. This sums the pulse waveforms outputted by other bonded cell body models.

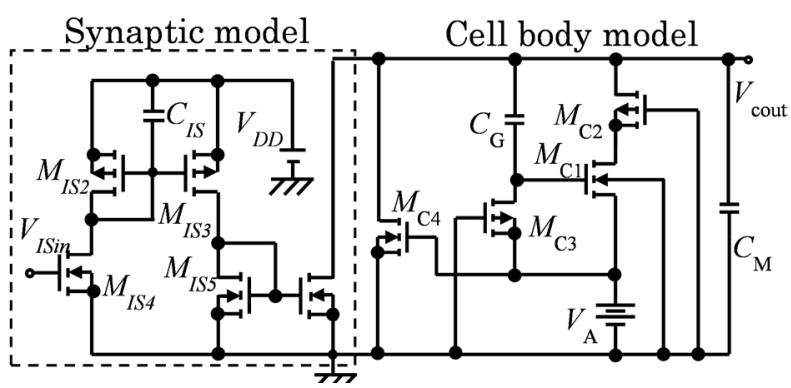

Fig. 7. Circuit diagram of the neuron.

\section{Results}

The bare chip IC of the neural networks made it possible for the control system to be mounted on the microrobot. Figure 8 shows the fabricated microrobot mounted the bare chip IC. The side, the end, and height dimensions of the fabricated PZT-type microrobot were 4.0, 4.6, and $3.6 \mathrm{~mm}$, respectively. The weight of the robot was $0.04 \mathrm{~g}$. The length and the width dimensions of the control circuit were $2.0,3.0 \mathrm{~mm}$, respectively. The side, the end, and height dimensions of the SMA-type microrobot were 4.0, 2.7, and $2.5 \mathrm{~mm}$, respectively. The weight of the robot was $0.02 \mathrm{~g}$. The length, width, and weight of the control circuit were $4.0 \mathrm{~mm}, 4.0 \mathrm{~mm}$, and $0.059 \mathrm{~g}$, respectively.

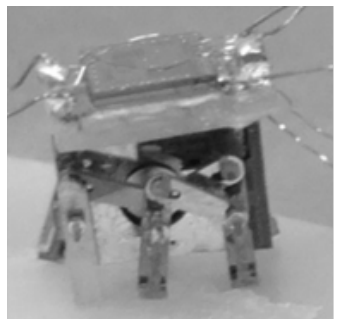

(a) PZT-type

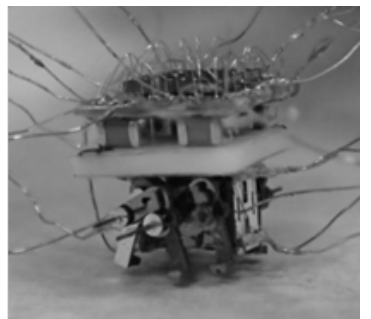

(b)SMA-type
Fig. 8. Fabricated microrobot mounted control system. (a) PZT-type (b) SMA-type.

PZT-type microrobot could generate the walking motion by the bare chip IC. However, the robot could not achieve locomotion by itself. Because, the bare chip IC is insufficient of the output voltage for moving legs. However, the bare chip IC for the PZT-type microrobot could drive the rotary actuator. Figure 9 shows the rotary motion of $1 \mathrm{~s}$ intervals. 


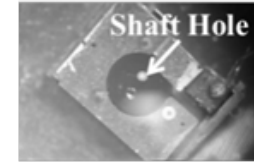

Os

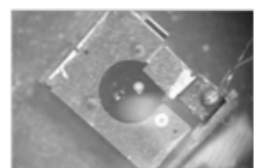

$3 \mathrm{~s}$

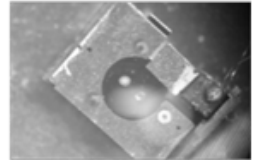

$1 \mathrm{~s}$

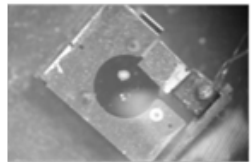

$4 \mathrm{~s}$

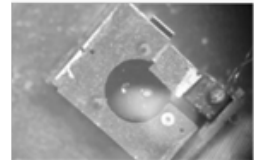

$2 \mathrm{~s}$

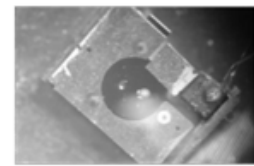

$5 \mathrm{~s}$
Fig. 9. Rotational motion of PZT-type actuator.

On the other hand, hexapod walking locomotion of the SMA-type microrobot, as shown in figure 10, was successfully achieved. The walking speed was 2.4 $\mathrm{mm} / \mathrm{min}$ and the step size was $0.083 \mathrm{~mm}$. Power source of the control circuit is externally connected.

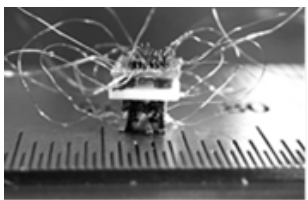

(a)

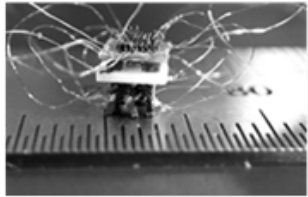

(c)

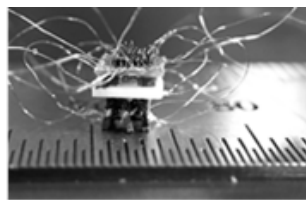

(b)

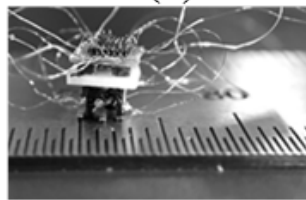

(d)
Fig. 10. Walking locomotion of the microrobot controlled by the bare chip IC. The figure show microrobot transitions at 10 $\mathrm{s}$ intervals.

\section{Conclusion}

In this paper, the control system of the neural networks was mounted the fabricated insect-type microrobot. It was possible to reduce the control system within a $4 \mathrm{~mm}$ square by using a bare chip IC. In the PZT-type microrobot, the bare chip IC could actuate the rotary actuator. Moreover, hexapod walking locomotion of the SMA-type microrobot was achieved. The walking speed was $2.4 \mathrm{~mm} / \mathrm{min}$ and the step size was $0.083 \mathrm{~mm}$. The only external wire connections to the microrobot are to the power source.

\section{Acknowledgements}

The MEMS microrobot fabrication was supported by the Research Center for Micro Functional Devices, Nihon University. This study was supported by Nihon University College of Science and Technology Project Research and JSPS KAKENHI (25420226). We appreciate the above support. The very large scale integration (VLSI) chip used in this study was fabricated by Digian Technology, Inc. This work was supported by the VLSI Design and Education Center (VDEC) of the University of Tokyo in collaboration with Synopsys, Inc., Cadence Design Systems, Inc. and Mentor Graphics, Inc.

\section{References}

1. A. T. Baisch, P. S. Sreetharan and R. J. Wood, Biologically-inspired locomotion of a $2 \mathrm{~g}$ hexapod robot, in Proc. 2010 Int. Conf. on Intelliigent Robots and systems, (Taipei, Republic of China, 2010), pp. 53605365.

2. B. R. Donald, C. G. Levey, C. D. McGray, I. Paprotny and D. Rus, An Untethered, Electrostatic, Globally Controllable MEMS Micro-Robot, J Microelectromechanical Systems. 15(1) (2006) 1-15.

3. S. A. Vukosavljev, D. Kukolj, I. Papp and B. Markoski, Mobile robot control using combined neuralfuzzy and neural network, in Proc. 2011 12th Int. Symp. on Computational Intelligence and Informatics, (Budapest, Hungary, 2011), pp. 351-356

4. M. Takato, M. Tatani, J. Tanida, S. Yamasaki, K. Saito, F. Uchikoba, Piezo Impact Type MEMS Rotary Actuator and Application to Millimeter Size AI Controlled Robot, in Proc. 2013 Int. Ultrasonic Symp., (Prague, Czech Republic, 2013), pp. 5360-5365.

5. K. Saito, Y. Sekine, M. Takato and F. Uchikoba, MEMS Microrobot with Pulse-Type Hardware Neural Networks Integrated Circuit, in Handbook of Research on Advancements in Robotics and Mechatronics, (Engineering Science Reference, Hershey PA, 2015), pp. 18-35.

6. K. Saito, Y. Ishihara, K. Sugita, Y. Okane, H. Oku, Y. Asano, K. Iwata, M. Tatani, M. Takato, Y. Sekine and F. Uchikoba, Artificial Neural Circuit Integration for MEMS Microrobot System, in Proc. 2015 Int. Conf. Advanced Intelligent Mechatronics, (Busan, Korea, 2015), pp. 1055-1060. 